\title{
Coherent heterodyne-assisted pulsed spectroscopy: sub-Doppler two-photon spectra of krypton, characterizing a tunable nonlinear-optical ultraviolet light source
}

\author{
Y. He • M. Kono • R.T. White • M.J. Sellars • \\ K.G.H. Baldwin • B.J. Orr
}

Received: 5 January 2010 / Revised version: 1 March 2010 / Published online: 14 May 2010

(c) Springer-Verlag 2010

\begin{abstract}
A chirp-minimized, nanosecond-pulsed system has been developed to generate tunable coherent ultraviolet light at $\sim 212.5 \mathrm{~nm}$ by fourth-harmonic conversion of output from an amplified, injection-seeded optical parametric oscillator (OPO). Our CHAPS (coherent heterodyneassisted pulsed spectroscopy) method is used to characterize the frequency stability and optical bandwidth of the system's output radiation by recording sub-Doppler twophoton excitation spectra of krypton. In our new scannedreference variant of CHAPS, the central frequency for each amplified OPO pulse is logged by the optical-heterodyne chirp-analysis software, with the frequency of the seed laser slowly tuned and monitored by a high-resolution wavemeter, unlike our previous implementation of CHAPS where the seed-laser frequency was fixed. For the amplified, upconverted pulses at $\sim 212.5 \mathrm{~nm}$, our CHAPS measurements indicate an optical bandwidth of $\sim 100 \mathrm{MHz}$, consistent with the Fourier-transform limit of their duration $(\sim 4.5 \mathrm{~ns})$.
\end{abstract}

Y. He · B.J. Orr $(\bowtie)$

MQ Photonics Research Centre, Department of Physics and Engineering, Macquarie University, Sydney, NSW 2109, Australia

e-mail: borr@physics.mq.edu.au

Fax: +61-2-9850-8289

M. Kono · M.J. Sellars · K.G.H. Baldwin

Research School of Physics and Engineering, The Australian

National University, Canberra, ACT 0200, Australia

R.T. White

Institute for Photonics and Advanced Sensing (IPAS), University

of Adelaide, Adelaide, SA 5005, Australia

\section{Introduction}

We have previously developed a high-performance all-solidstate nonlinear-optical (NLO) light source to produce narrowband tunable pulses of coherent light in the range of $820-850 \mathrm{~nm}$ with $\leq 5 \mu \mathrm{J}$ energy, as a versatile source of tunable single-longitudinal-mode (SLM) coherent light for high-resolution spectroscopy [1-7]. It is useful in applications for which high peak power and narrow optical bandwidth are needed (e.g., in tens-of-ns pulses), especially in the vacuum ultraviolet (VUV) region where NLO upconversion is essential.

For instance, our system will generate narrowband VUV light at $\sim 120 \mathrm{~nm}$ (by frequency-quadrupling light at $\sim 840$ to $\sim 210 \mathrm{~nm}$, then two-photon-resonant four-wave mixing in krypton, $\mathrm{Kr}$ ) to measure the $2{ }^{1} \mathrm{~S} \leftarrow 1{ }^{1} \mathrm{~S}$ two-photon transition of helium (He) as a test of quantum-electrodynamic theory. Previous studies, using a continuous-wave $(\mathrm{cw})$ tunable Ti:sapphire laser and a pulsed dye amplifier [8-10], yielded limited bandwidth and uncertainties attributable to population-inversion and refractive-index variations in the dye amplifier. We expect our new NLO VUV light source to circumvent such problems.

We use a high-performance pulsed optical parametric oscillator (OPO) based on periodically poled $\mathrm{KTiOPO}_{4}$ (PPKTP), pumped by a long-duration ( $27 \mathrm{~ns})$ pulsed SLM Nd:YAG laser (Continuum Powerlite 9000) and injectionseeded by a cw Ti:sapphire ring laser (Coherent 899-21) $[5,6]$, to generate tunable SLM coherent light in the wavelength range of $820-850 \mathrm{~nm}$. Optical amplification and fourth-harmonic generation $(4 \mathrm{HG})$ then yield up-converted ultraviolet pulsed radiation. To validate the spectroscopic utility of this all-solid-state tunable SLM source of coherent pulsed ultraviolet radiation, we measure the sub-Doppler spectral profile of a two-photon resonance in $\mathrm{Kr}$ gas at $\sim 212.55 \mathrm{~nm}$. 


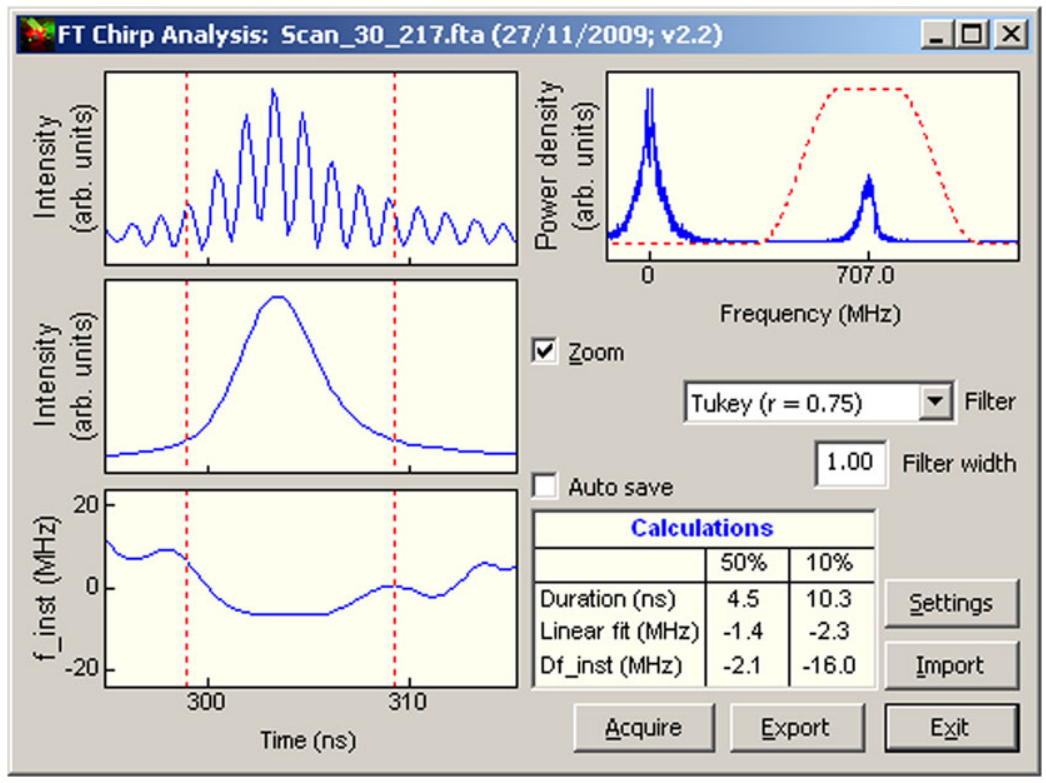

Fig. 1 Optical-heterodyne-detected chirp-analysis software [7] provides a real-time display of the recorded beat waveform (top, left) and its Fourier transform (with filter function; top and center, right), the reconstructed profile of the amplified OPO signal pulse (center, left), the calculated instantaneous-frequency profile (bottom, left), and cal-

As previously reported [3-7], the primary OPO signal output at $\sim 850 \mathrm{~nm}$ has well-characterized shot-to-shot frequency stability and minimal frequency chirp, with its optical bandwidth approaching the sub-20-MHz Fouriertransform limit for a pulse duration of $\sim 25 \mathrm{~ns}$. An opticalheterodyne detection (OHD) technique [1-7] is able to monitor frequency chirp, fluctuations in the temporal profiles, and central-frequency shifts for successive optical pulses, thereby minimizing the spectroscopic limitations of such operating characteristics.

Sub-Doppler spectroscopic measurements play a key role in verifying the performance of this pulsed tunable NLO system at successive stages of its operation. For example, the fundamental OPO signal output has previously been characterized via sub-Doppler two-photon $8 \mathrm{~S} \leftarrow 6 \mathrm{~S}$ spectra of cesium (Cs) vapor at $\sim 822.5 \mathrm{~nm}[5,6]$. To do this, we devised the CHAPS (Coherent Heterodyne-Assisted Pulsed Spectroscopy) approach, with the reference frequency of the $\mathrm{cw}$ Ti:sapphire seed laser held fixed on a Cs resonance peak and our OHD method used to $\log$ the central frequency of each OPO pulse (pre- or post-OPA) and record a sub-Doppler spectrum spanned by the OPO or OPA frequency jitter.

We report a novel scanned-reference variant of CHAPS, in which the cw seed-laser frequency is slowly tuned and monitored by a high-resolution wavemeter. By controlled tuning of the $\mathrm{cw}$ seed laser and logging the central frequency for each amplified OPO pulse at $\sim 850.2 \mathrm{~nm}$ by means of our OHD real-time chirp-analysis software, we can correct any pulse-to-pulse OPO or amplifier frequency fluctuations and culated pulse durations and chirp parameters (bottom right). In the example shown, the OPO is well seeded with frequency chirp $<10 \mathrm{MHz}$ (between 50\% intensity points-table, lower right). It is remarkable that optical amplification does not cause the frequency chirp to deteriorate

produce sub-Doppler CHAPS spectra of the $4 \mathrm{p}^{5} 5 \mathrm{p}[1 / 2]_{0} \leftarrow$ $4 \mathrm{p}^{6}$ two-photon resonance in $\mathrm{Kr}$ at $\sim 212.55 \mathrm{~nm}$ [11-13], over a frequency range exceeding that spanned by frequency jitter of the pulsed OPO.

\section{Experimental}

To amplify the OPO output at $\sim 850 \mathrm{~nm}$ prior to NLO upconversion, we previously used a $\mathrm{LiNbO}_{3}$ optical parametric amplifier (OPA). This OPA provided a pulse-energy gain of $\sim 8$, but the optical pulse duration was reduced from $\sim 23$ to $\sim 15$ ns FWHM, degrading the Fourier-transform-limited optical bandwidth [5, 6]. To achieve much higher optical power $(\sim 8000 \times$ gain), we have installed an advanced twostage Ti:sapphire amplifier (Amplitude Technologies), emulating recent work [14] on amplification of SLM Ti:sapphire laser output. The booster and multipass stages of this amplifier (pumped at $532 \mathrm{~nm}$ by $\sim 35-\mathrm{mJ}$ and $\sim 200-\mathrm{mJ}$ pulses from our long-pulse Nd:YAG laser) increase the SLM OPO signal output pulse energies from $\leq 5 \mu \mathrm{J}$ to $\sim 1 \mathrm{~mJ}$ and $\sim 40 \mathrm{~mJ}$, respectively, with good spatial beam profile.

As in previous work [1-7], we measure OHD beats between amplified OPO pulses and cw light from the seed laser (modulator-shifted by $\sim 730 \mathrm{MHz}$ ), to characterize frequency-chirp effects due to time-dependent optical phase variations during the OPO pulse and in subsequent amplification stages. We have previously shown [1-7] how to 


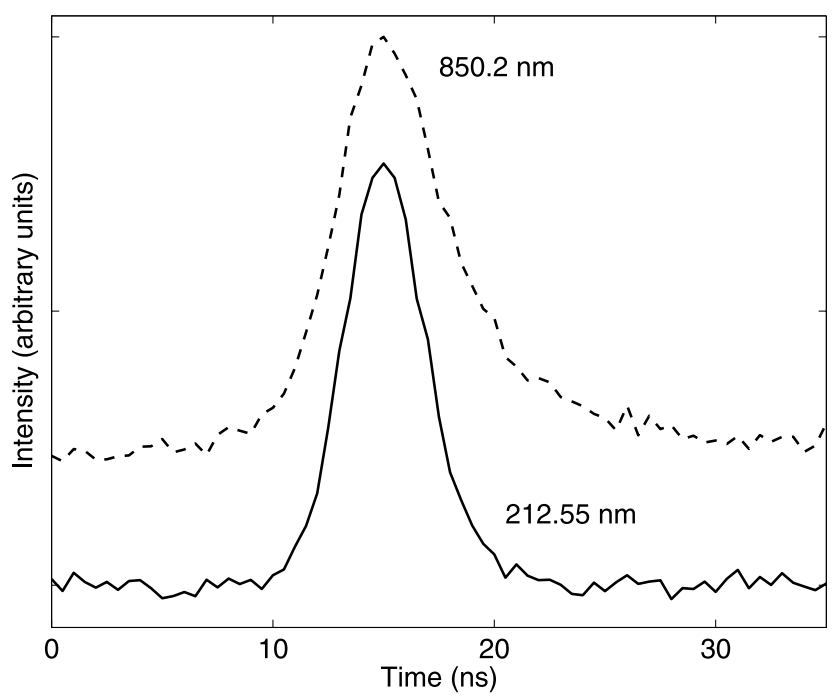

Fig. 2 Temporal profiles of amplified fundamental and up-converted 4HG pulses (at $\sim 850.2 \mathrm{~nm}$ and $\sim 212.55 \mathrm{~nm}$, respectively), measured by a fast photodetector (Alphalas, model UPD-200-UD, 2.0-GHz bandwidth, <0.2-ns rise-time)

minimize frequency chirp by operating the OPO system at its phase-matching wavelength (when the free-running OPO and seed wavelengths coincide). Comparable measures have been adopted to optimize instantaneous-frequency characteristics of the Ti:sapphire amplifier output, as depicted in Fig. 1, which is a representative frame generated by our OHD software. The chirp is remarkably small (well below $10 \mathrm{MHz}$ FWHM) but the amplified pulse duration is disappointingly short ( $\leq 6 \mathrm{~ns}$ FWHM, compared with $\sim 25 \mathrm{~ns}$ for the OPO output pulse itself). This four-fold opticalbandwidth deterioration seems to arise from extraction of most of the gain of the Ti:sapphire pulsed amplifier by the leading part of each OPO pulse; this problem is being addressed in pursuit of higher spectroscopic resolution.

The SLM pulsed output at $\sim 850.2 \mathrm{~nm}$ from the Ti: sapphire-amplified OPO is up-converted by $4 \mathrm{HG}$ to $\sim 212.55 \mathrm{~nm}$ in two second-harmonic-generation crystals: $\mathrm{LiB}_{3} \mathrm{O}_{5}$, followed by $\beta-\mathrm{BaB}_{2} \mathrm{O}_{4}$. A pulse energy of $\sim 40 \mathrm{~mJ}$ at $\sim 850.2 \mathrm{~nm}$ yields $\sim 1 \mathrm{~mJ} /$ pulse at $\sim 212.55 \mathrm{~nm}$. Temporal profiles of fundamental and 4HG pulses, measured by a 2.0-GHz photodetector, are shown in Fig. 2; their FWHM durations are $\sim 6$ and $\sim 4.5 \mathrm{~ns}$, respectively. The profile of the pulse at $\sim 212.55 \mathrm{~nm}$ is quasi-Gaussian; its corresponding Fourier-transform-limited optical bandwidth would be $\sim 100 \mathrm{MHz}$.

The pulsed coherent light at $\sim 212.55 \mathrm{~nm}(\sim 3-\mathrm{mm}$ diameter) propagates to the $\mathrm{Kr}$ ionization cell, where it is collimated and $3 \times$-expanded by a two-lens Galilean telescope. This parallel, unfocused beam is retro-reflected back through the cell by a flat mirror. With pure $\mathrm{Kr}$ gas in the cell, the two-photon-resonant ionization signal is collected by a longitudinal electrode with a DC bias voltage of $36 \mathrm{~V}$ and a
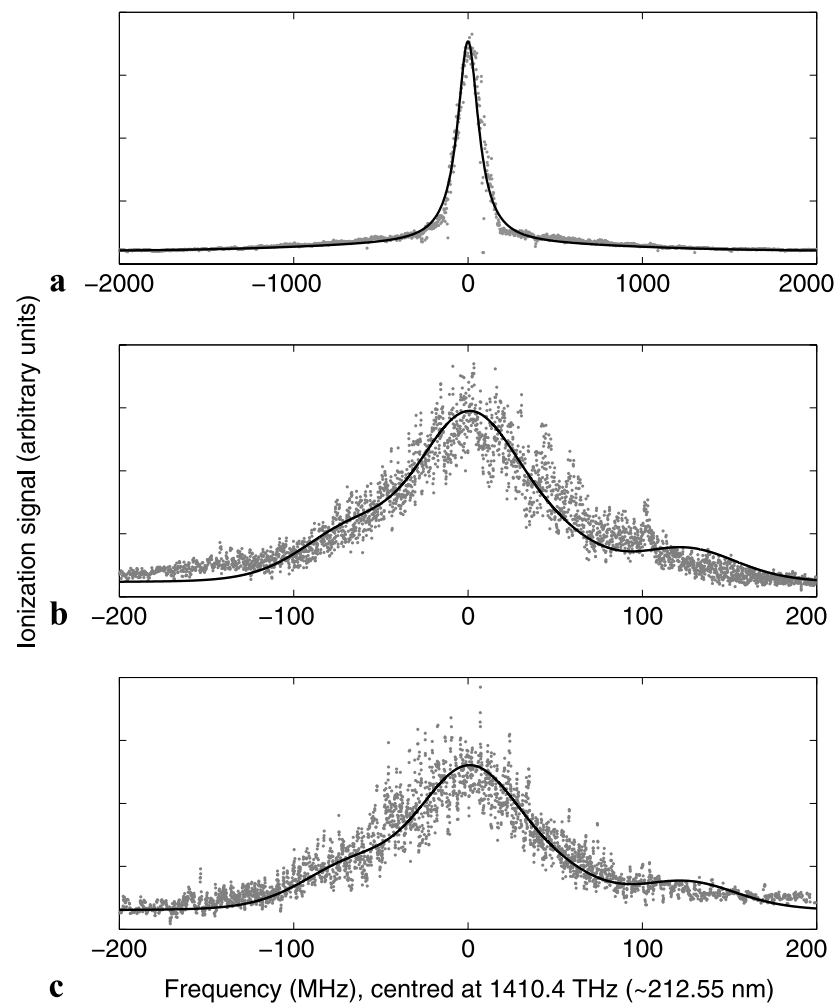

Fig. 3 Two-photon-resonant ionization spectra of the $4 \mathrm{p}^{5} 5 \mathrm{p}[1 / 2]_{0} \leftarrow 4 \mathrm{p}^{6}$ transition in $\mathrm{Kr}$ gas, recorded by means of our chirp-minimized narrowband tunable source of coherent ultraviolet light at $\sim 212.55 \mathrm{~nm}$ : (a) a wide-ranging scan of the spectral profile, comprising a sub-Doppler peak ( $\sim 150-\mathrm{MHz}$ FWHM) superimposed upon a Doppler-broadened pedestal ( $\sim 2-\mathrm{THz}$ FWHM); (b) a more detailed scan of the sub-Doppler peak with the boxcar-detected ionization signal plotted without correcting for frequency fluctuations in the amplified OPO pulse; (c) data corresponding to trace (b), following CHAPS analysis to determine the central frequency of each pulse relative to the (tuned) $\mathrm{cw}$ Ti:sapphire seed-laser frequency. The pulsed optical power is typically $<0.1 \mathrm{MW} \mathrm{cm}^{-2}$ and $\mathrm{Kr}$ pressures are: (a) 0.13 Torr (17 Pa); (b, c) 0.10 Torr (13 Pa). Superimposed on traces (b) and (c) is a tentatively simulated spectrum, based on known $\mathrm{Kr}$ isotopic shifts and abundances $[14,15]$

boxcar integrator to record survey spectra. The $\mathrm{Kr}$ pressure and incident optical power (varied by a polarizing attenuator) were carefully optimized to record reliable, saturationfree two-photon-resonant ionization signals.

\section{Results and discussion}

Figure 3 shows spectra obtained for the $4 \mathrm{p}^{5} 5 \mathrm{p}[1 / 2]_{0} \leftarrow 4 \mathrm{p}^{6}$ two-photon-resonant ionization process in $\mathrm{Kr}$ gas, as a function of the tuned $4 \mathrm{HG}$ frequency (four times the frequency of the amplified OPO signal output). The broad spectral profile in Fig. 3(a) shows the Doppler-broadened pedestal on which the sub-Doppler peak is superimposed. Figures 3(b) and 3(c) are more detailed scans of the sub-Doppler peak, without 
and with CHAPS-type corrections for frequency fluctuations in the amplified OPO pulse, respectively.

The synthetic spectrum, superimposed in Figs. 3(b) and 3(c), takes account of $\mathrm{Kr}$ isotopic components that are separated by intervals of $50-80 \mathrm{MHz}$ on the $4 \mathrm{HG}$ frequency scale $[14,15]$, with relative intensities that reflect the natural abundance of the major isotopes of $\mathrm{Kr}\left(57.0 \%{ }^{84} \mathrm{Kr}, 17.3 \%\right.$ ${ }^{86} \mathrm{Kr}, 11.6 \%{ }^{82} \mathrm{Kr}, 11.5 \%{ }^{83} \mathrm{Kr}$ ) but do not allow for possible isotopic variation in the two-photon transition probability. The simulation allows crudely for the spectral bandwidth $(\sim 100 \mathrm{MHz})$ derived from the pulse duration $(\sim 4.5-\mathrm{ns})$ and the underlying Doppler pedestal from Fig. 3(a), but neglects pressure broadening ( $\leq 5 \mathrm{MHz}$ FWHM [16]).

The $\sim 100-\mathrm{MHz}$ FWHM linewidth of the coherent light at $\sim 212.55 \mathrm{~nm}$ is limited predominantly by the $\sim 4.5-\mathrm{ns}$ optical pulse duration and does not allow clear resolution of the $\mathrm{Kr}$ isotopic structure in the $4 \mathrm{p}^{5} 5 \mathrm{p}[1 / 2]_{0} \leftarrow 4 \mathrm{p}^{6}$ two-photon excitation spectrum. We note that these spectra were recorded with minimal frequency chirp $(\leq 10 \mathrm{MHz}$ at $\sim 850 \mathrm{~nm})$ and with small shot-to-shot fluctuations ( $\sim 4$ $\mathrm{MHz}$ standard deviation at $\sim 850 \mathrm{~nm}$ ) in the central frequency. The fit of the simulated curve to the CHAPSanalyzed data in Fig. 3(c) is therefore not markedly superior to that for the boxcar-detected data in Fig. 3(b).

\section{Conclusion}

We have measured two-photon-resonant ionization spectra of $\mathrm{Kr}$ at $\sim 212.55 \mathrm{~nm}$ to characterize a nearly Fouriertransform-limited OPO light source following amplification and 4HG frequency conversion, using a new scannedreference variant of CHAPS. The spectra obtained are consistent with the optical bandwidth and shot-to-shot frequency stability determined by optical-heterodyne measurements, which indicate that the linewidth is dominated by the relatively short pulse duration ( $\sim 4.5 \mathrm{~ns}$ FWHM). In ongoing research, we intend to circumvent the Ti:sapphire amplifier system's severe pulse-shortening effect and attain significantly higher sub-Doppler spectroscopic resolution.

Our progress towards measurement of the fundamentally important $2{ }^{1} \mathrm{~S} \leftarrow 1{ }^{1} \mathrm{~S}$ two-photon spectrum of He by generating $\sim 120$-nm tunable SLM coherent light (enhanced by the same two-photon resonance in $\mathrm{Kr}$ ) complements recent advances elsewhere [13, 14, 17, 18], aiming to address discrepancies between state-of-the-art theory $[19,20]$ and earlier experimental estimates $[8,21]$ of the Lamb shift in ground-state He.

Acknowledgements We acknowledge funding from the Australian Research Council and thank Drs S. Cavanagh and W. Krolikowski for experimental assistance. The Ti:sapphire amplifier system used was custom-built for us by Amplitude Technologies (France).

\section{References}

1. R.T. White, Y. He, B.J. Orr, M. Kono, K.G.H. Baldwin, Opt. Lett. 28, 1248 (2003)

2. R.T. White, Y. He, B.J. Orr, M. Kono, K.G.H. Baldwin, J. Opt. Soc. Am. B 21, 1577 (2004)

3. R.T. White, Y. He, B.J. Orr, M. Kono, K.G.H. Baldwin, J. Opt. Soc. Am. B 21, 1586 (2004)

4. R.T. White, Y. He, B.J. Orr, M. Kono, K.G.H. Baldwin, Opt. Express 12, 5655 (2004)

5. M. Kono, K.G.H. Baldwin, Y. He, R.T. White, B.J. Orr, Opt. Lett. 30, $3413(2005)$

6. M. Kono, K.G.H. Baldwin, Y. He, R.T. White, B.J. Orr, J. Opt. Soc. Am. B 23, 1181 (2006)

7. R.T. White, Y. He, B.J. Orr, M. Kono, K.G.H. Baldwin, J. Opt. Soc. Am. B 24, 2601 (2007)

8. S.D. Bergeson, A. Balakrishnan, K.G.H. Baldwin, T.B. Lucatorto, J.P. Marangos, T.J. McIlrath, T.R. O’Brian, S.L. Rolston, C.J. Sansonetti, J. Wen, N. Westbrook, C.H. Cheng, E.E. Eyler, Phys. Rev. Lett. 80, 3475 (1998)

9. S.D. Bergeson, A. Balakrishnan, K.G.H. Baldwin, T.B. Lucatorto, J.P. Marangos, T.J. McIlrath, T.R. O’Brian, S.L. Rolston, C.J. Sansonetti, J. Wen, N. Westbrook, C.H. Cheng, E.E. Eyler, Phys. Scr., T 83, 76 (1999)

10. S.D. Bergeson, K.G.H. Baldwin, T.B. Lucatorto, T.J. McIlrath, C.H. Cheng, E.E. Eyler, J. Opt. Soc. Am. B 17, 1599 (2000)

11. J.P. Marangos, N. Shen, H. Ma, M.H.R. Hutchinson, J.P. Connerade, J. Opt. Soc. Am. B 307, 400 (2005)

12. F. Brandi, W. Hogervorst, W. Ubachs, J. Phys. B: At. Mol. Opt. Phys. 35, 1071 (2002)

13. S. Witte, R.T. Zinkstok, W. Ubachs, W. Hogervorst, K.S.E. Eikema, Science 307, 400 (2005)

14. S. Hannemann, E.-J. van Duijn, W. Ubachs, Rev. Sci. Instrum. 78, 103102 (2007)

15. F. Brandi, W. Hogervorst, W. Ubachs, J. Phys. B: At. Mol. Opt. Phys. 35, 1071 (2002)

16. D.A. Jackson, J. Opt. Soc. Am. 71, 1064 (1981)

17. E.E. Eyler, D.E. Chieda, M.C. Stowe, M.J. Thorpe, T.R. Schibli, J. Ye, Eur. Phys. J. D 48, 43 (2008)

18. K.S.E. Eikema, Private communication, Vrije Universiteit, Amsterdam, 2009

19. K. Pachucki, Phys. Rev. A 74, 022512 (2006)

20. D.C. Morton, Q. Wu, G.W.F. Drake, Canad. J. Phys. 84, 83 (2006)

21. K.S.E. Eikema, W. Ubachs, W. Vassen, W. Hogervorst, Phys. Rev. A 5, 1866 (1997) 